\title{
Characterization of Neutron and Gamma Beams at the TRIGA 2000 Reactor Beam Ports Using Monte Carlo
}

\author{
Rasito $^{1,2^{*}}$, S. Permana ${ }^{1}$, and P. Ilham Y. ${ }^{1}$ \\ ${ }^{1}$ Nuclear Physics Laboratory, Physics Department, Bandung Institute of Technology, Jl. Ganeca 10, Bandung \\ ${ }^{2}$ Center for Science and Applied Nuclear Technology, National Nuclear Energy Agency, Jl. Tamansari 71, Bandung
}

( Received: August 28, 2018, Revised: December 13, 2018, Accepted: August 7, 2019 )

\begin{abstract}
Neutron and gamma beams characterization at the beam ports of TRIGA 2000 reactor was done in order to prepare the development of Prompt Gamma Neutron Activation Analysis (PGNAA) facility. Characterization was performed by simulation using Monte Carlo method with MCNPX and PHITS computer codes. The MCNPX code is used to simulate the neutron-gamma fluence and spectra of the reactor core from fission reactions. The PHITS code is used to simulate the distribution of the neutron-gamma fluence in the beam ports. The simulation was done by modeling the geometry and elemental composition of reactor material and radiation source model in the form of fission reaction in the reactor core. This results will be used to select the most suitable one of the four beam ports for the PGNAA use, based on the prescribed requirements, such as the neutron spectral characteristics and neutron-gamma fluence ratio at the beam port's outlet. The results indicate that the tangential beam port provides better characteristics of neutron spectrum and neutrongamma fluence ratio so become most suitable for PGNAA facility.
\end{abstract}

Keywords: Neutron-gamma, Beam ports, Triga Reactor, MCNPX, PHITS

\section{INTRODUCTION}

The PGNAA technique or also called gamma activation analysis (PGAA) is a technique for analyzing elements based on gamma rays emitted directly from a sample due to interactions with neutrons. In its application this technique requires a source of neutrons in the form of radioactive sources and neutron generators. One source of neutrons that can be used is a nuclear reactor. The large neutron flux from a nuclear reactor allows it to be used for PGNAA.

Some of TRIGA type reactors have been used for PGAA facilities utilizing beam ports, such as TRIGA III reactors in Mexico with a neutron flux of $7 \times 10^{7} \mathrm{n} / \mathrm{cm}^{2} \mathrm{~s}$ and a dose of $0.1 \mathrm{~Sv} / \mathrm{j}$ gamma [1]. There were also those who designed additional beam ports by passing them to primary cooling water as in Brazil [2]. To be able to use the reactor beam beam as a neutron source for PGNAA, it is necessary to do flux characterization and neutron spectrum and gamma output beam port [3][4].

\footnotetext{
* Corresponding author.

E-mail address: rasito@batan.go.id
}

Determination of the neutron-gamma spectrum by simulation was done using a computer program based on the Monte Carlo method. The Monte Carlo method is a numerical statistical method that simulates random numbers to solve to solve problems that are difficult to solve using analytical methods. The computer program based on the Monte Carlo method used to simulate neutrongamma in the reactor beam port is MCNPX and PHITS. The MCNPX computer program is a Monte Carlo-based computer software that was applied to calculate the transport of neutron particles, photons, and criticality, etc. [5]. The PHITS code is a computer program based on Monte Carlo method developed by Japan Atomic Energy Agency (JAEA) which has the ability to simulate the journey of neutron particles, photons, etc. with a wide range of energy [6].

In order to utilize the TRIGA 2000 reactor, BATAN has plans to build PGNAA facilities by utilizing the neutron beam output from the beam port. This simulation results will be used to select the most suitable one of the four beam ports for the PGNAA use, based on the prescribed requirements, 
such as the neutron spectral characteristics and neutron-gamma fluence ratio at the beam port's outlet. How to characterize neutron and gamma beams and also the energy spectrum in the beam ports using MCNPX and PHITS then will be described in this paper.

\section{METHODS}

Flux and neutron-gamma spectrum simulations at tangential beam port of the TRIGA 2000 reactor was done using Monte Carlo method with MCNPX and PHITS computer codes. The MCNPX program was used to determine the flux and neutron-gamma spectrum resulting from fission reactions on the reactor core. The neutron and gamma spectra on the reactor core were used as radiation sources for PHITS inputs. The PHITS code was used to simulate the flux distribution and neutron-gamma spectrum in the beam ports.

\section{Neutron-Gamma TRIGA core}

The first stage is the flux simulation and neutron-gamma spectrum on the reactor core using the MCNPX code. This MCNPX code was used because it has been tested for its ability in reactor criticality calculations supported by complete neutron interaction cross-sections data [7][8]. To run the MCNPX code requires inputs; reactor geometry, reactor material composition, radiation source model, and tally flux. TRIGA 2000 reactor has a complex geometry so it is quite difficult in modeling. The model of reactor geometry is covering the reactor core, reflector, and cooling water as shown in Fig. 1. The shape and size of the reactor part was made by a geometry model in the MCNPX input. Material data and cross section of each reactor component are also made for MCNPX input. The materials used are water, aluminum, SS 304, boron carbide, UZr-H fuel elements, zirconium, and graphite.

The next MCNPX input is a radiation source model. Radiation in a nuclear reactor comes from the fission reaction of a ${ }^{235} \mathrm{U}$ fuel with thermal neutrons. In each fission reaction, a ${ }^{235} \mathrm{U}$ nucleus produced $180 \mathrm{MeV}$ of energy so that for a TRIGA 2000 reactor operating at $2 \mathrm{MW}$, there were $7 \times 10^{16}$ fission reactions per second. Each fission reaction will produce an average of 2.4 neutrons, so in the reactor core there is $1.68 \times 10^{17} \mathrm{n} / \mathrm{s}$. Flux modeling was done to get the MCNPX output in the form of flux values on the reactor core. In this modeling several types of tally were used including tally energy (E4), tally flux for neutrons, F4: $\mathrm{n}$ and for photons, (F4: p). Tally E4 is the energy bin that is determined to display the value of flux in a certain energy range. With the tallies, MCNPX will give output in the form of neutron and gamma energy spectrum [5].

\section{Neutron-Gamma at the Beam Ports}

The second stage is simulation for flux and neutron-gamma spectrum in the beam ports using PHITS code. The PHITS input consists of reactor geometry, beam ports, and material data. Some of the materials used in the input include graphite, aluminum, SS 304, concrete, and air. The radiation source that was modeled is a cylindrical surface (reactor core) with a diameter of $25.88 \mathrm{~cm}$ and a height of $60 \mathrm{~cm}$. Flux value and energy source of the radiation source were taken from the MCNPX simulation results.

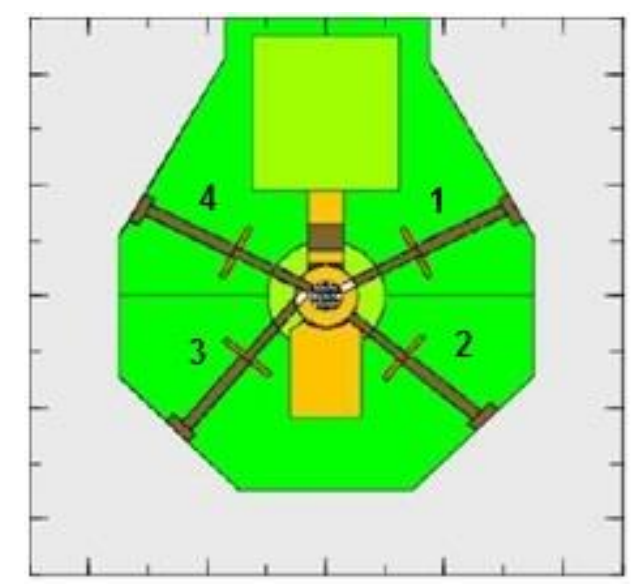

Figure 1. Beam ports of TRIGA 2000 reactor

In this second simulation, the TRIGA 2000 reactor core became a neutron-gamma source. So that the reactor core geometry was converted into cylinders with no material. To get PHITS output in the form of neutron-gamma flux distribution in the beam port and spectrum at the end of the beam port, tally and t-cross tally are used. Tally t-track will produce a neutron-gamma flux distribution in the beam port according to the specified grid, while tcross produces an energy distribution in the object (detector) at the end of the beam port.

\section{RESULTS AND DISCUSSION}

Flux distribution and neutron-gamma energy spectrum have been simulated using MCNPX. The MCNPX program is run on a computer with an Intel Core i5- $2.3 \mathrm{GHz}$ processor, 4GB RAM, and Windows 8.1 operating system. In the simulation used data on neutron latitude from ENDF-VI.2, gamma production using NJOY, and atomic photo interaction using ENDF/B-VI. With these computer specifications, it takes 100 minutes of simulation 
time for KCODE with 100000 initial neutrons, 250 effective cycles and 50 ineffective cycles has been used for the parameter calculations.

Neutron and gamma flux values vary according to position on the reactor core. The more the position to the center, the greater the flux. The distribution of neutron flux in the reactor core ranges from $10^{13-} 10^{14} \mathrm{~cm}^{-2} \cdot \mathrm{s}^{-1}$. The neutron energy spectrum was dominated by thermal neutrons with energies below $0.5 \mathrm{eV}$. The gamma energy spectrum was dominated by high energy gamma which is 1 $10 \mathrm{MeV}$. The neutron flux at the position of the reactor core wall is $10^{12} \mathrm{~cm}^{-2} \cdot \mathrm{s}^{-1}$. The distribution of neutron and gamma fluxes along with their energy spectrum are then used as new source definitions in simulating flux distribution and neutron-gamma energy in tangential port beams using the PHITS code.

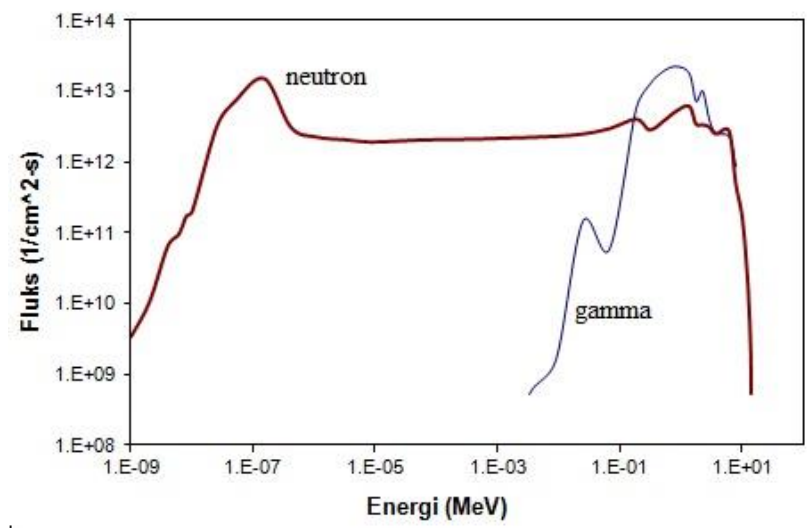

Figure 2. Neutron-gamma spectrums in the TRIGA 2000 reactor core

The PHITS code was used to simulate the passage of neutron and gamma particles from the reactor core to the end of beam ports. The reactor core produces neutrons and gamma which are radiated isotropically. Most neutrons and gamma were absorbed and reflected by graphite reflector walls. In Fig. 3 shows the neutron flux distribution at the end of the beam ports, and Fig. 4 shows the gamma flux distribution.

Neutron flux at the end of the beam port 1 and 4 is about $10^{8} \mathrm{~cm}^{-2} \cdot \mathrm{s}^{-1}$, while beam port 2 and 3 is about $10^{7} \mathrm{~cm}^{-2} \cdot \mathrm{s}^{-1}$. Gamma flux at the end of the beam port 1 is about $10^{6} \mathrm{~cm}^{-2} \cdot \mathrm{s}^{-1}$, while the beamport 2,3 , and 4 about $5 \times 10^{6} \mathrm{~cm}^{-2} \cdot \mathrm{s}^{-1}$. The diameter of the field at the end of the beam port is $20 \mathrm{~cm}$, and at a distance of $1 \mathrm{~m}$ from the end of the beam port the diameter of the beam is $40 \mathrm{~cm}$. the flux value varies with the highest flux in the center of the field. To get a large flux, it can be done by placing an additional cone-shaped collimator with material that has the characteristics of reflecting neutrons.

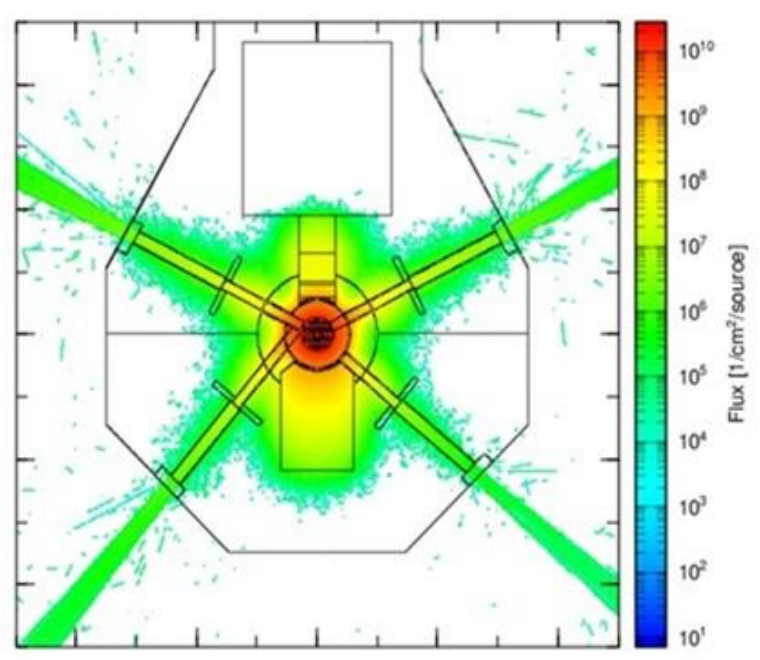

Figure 3. Neutron flux at the beam ports

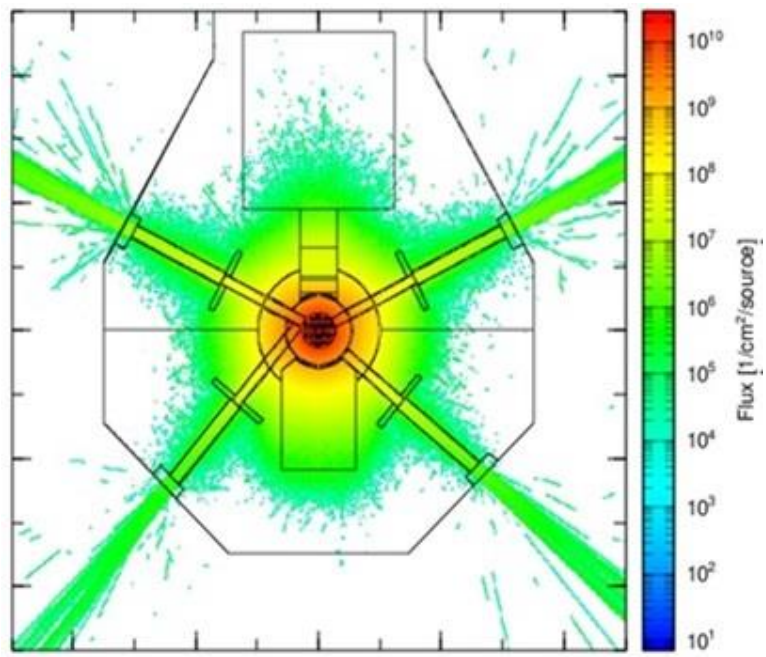

Figure 4. Gamma flux at the beam ports

The results of the PHITS simulation to determine the neutron and gamma energy spectrum at the end of the beam ports was shown in Fig. 3 and 4. The neutron ratio of thermal-fast neutrons at the end of the beam port 1 is 7.6 (Fig. 5), beam port 2 is 21 (Fig. 6), beam port 3 is 14.4 (Fig. 7), and beam port 4 is 3.3 (Fig. 8). Gamma flux ratio with neutrons at the end of the beam port 1 is 2.9 (Fig. 5), beam port 2 is 2.0 (Fig. 6), beam port 3 is 2.2 (Fig. 7), and beam port 4 is 1.5 (Fig. 8). The largest fastthermal neutron ratio is generated from the second radial beam port, which is 21 . However, this beam port also produces a large gamma-neutron ratio of 0.5 . The lowest gamma-neutron ratio is generated from the first radial port beam which is 0.34 . But this beam port produces a low thermal neutron ratio, which is also low, which is 7.6. 


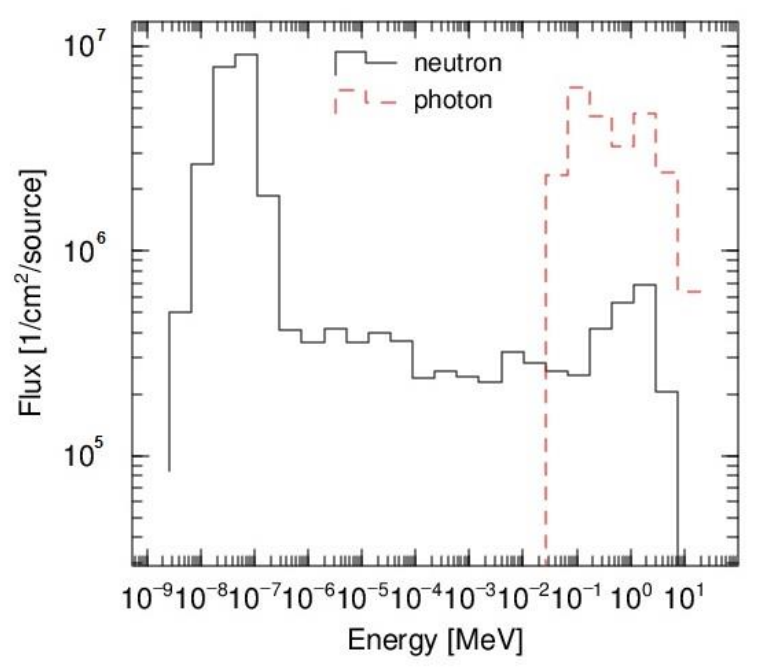

Figure 5. Neutron-gamma spectra at beam port 1

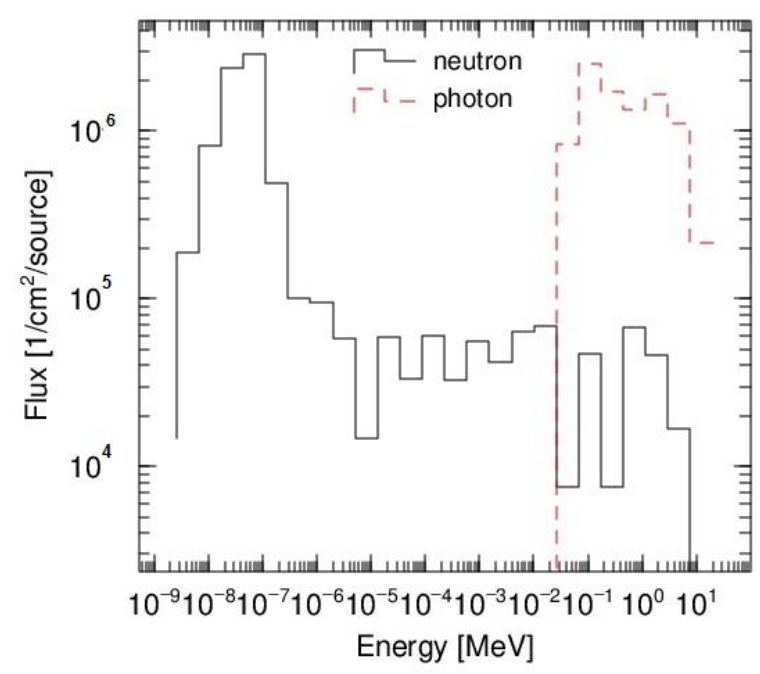

Figure 6. Neutron-gamma spectra at beam port 2

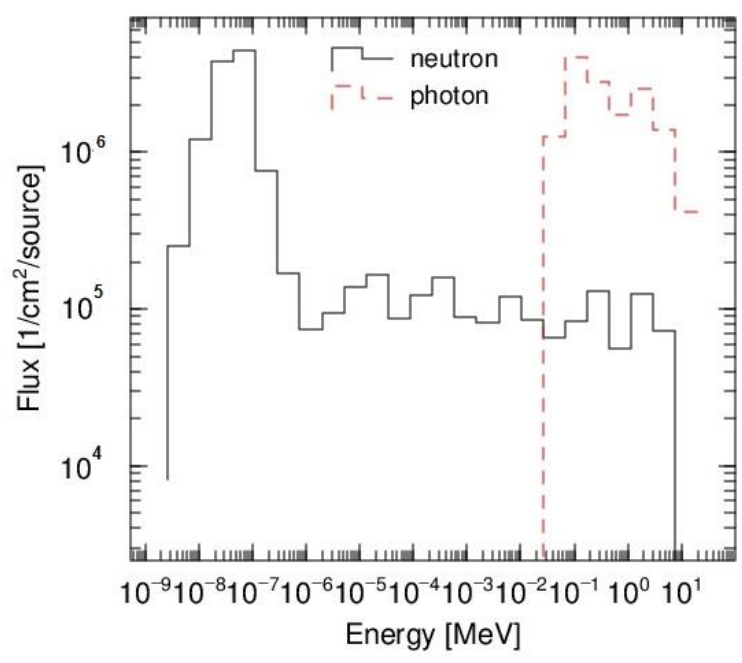

Figure 7. Neutron-gamma spectra at beam port 3

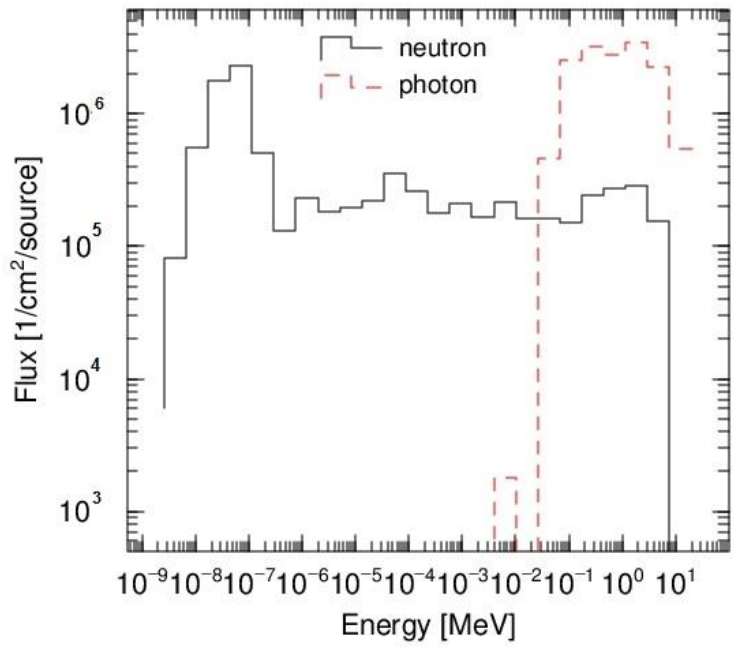

Figure 8 . Neutron-gamma spectra at beam port 4

The use of TRIGA reactor beam ports for PGNAA facilities can be done by considering several things, including high neutron flux, high thermal-fast neutron ratio, and low gamma flux. Tangential beam port (beam port 3) were the most ideal for use as PGNAA facilities. The choice of beam port with high thermal neutron flux will be advantageous because the sample irradiation time will be shorter. The selection of the beam ports with low gamma flux will be beneficial, ie the background gamma radiation will be low. So it doesn't need a thick gamma shield design.

\section{CONCLUSION}

The MCNPX program has been used well to model the flux and neutron-gamma spectrum on the TRIGA 2000 reactor core. At power of $2 \mathrm{MW}$ the

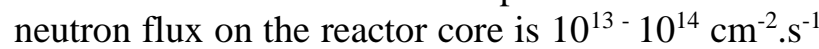
which is dominated by thermal neutrons $\left(>5 \times 10^{-7}\right.$ $\mathrm{MeV}$ ) and gamma energy 1-10 MeV. The PHITS program has been used well to model the flux and neutron-gamma spectrum at the tangential beam port of TRIGA 2000 reactor. At power of $2 \mathrm{MW}$ the neutron flux at the end of the tangential port beam is $10^{7} \mathrm{~cm}^{-2} \cdot \mathrm{s}^{-1}$. The selection of beam ports for PGNAA facilities with the criteria of high thermalfast neutron and low gamma-neutron ratio, the tangential port beam be the best choice.

\section{ACKNOWLEDGMENT}

The authors gratefully acknowledge to the support given to this work from joint cooperation between National Nuclear Energy Agency 
(BATAN) and Bandung Institute of Technology (ITB). Also for reactor division and safety division of the Center for Science and Applied Nuclear Technology (PSTNT).

\section{REFERENCES}

[1] Rios-Martinez, C., Paredes-Gutierrez, L., Arias, E. Alemon, Ortiz-Romero, M.E., 2001, Design of a PGAA Facility at the TRIGA Mark III of ININ, Mexico, 2001 Annual meeting; Milwaukee, WI (United States)

[2] Bruno $\mathrm{T}$ Guerra, Radojko Jacimovic, Maria Angela BC Menezes, dan Alexandre S Leal, 2013, Proposed design for the PGAA facility at the TRIGA IPR-R1 research reactor, SpringerPlus, 2,:597, http://www.springerplus.com/content/2/1/597

[3] Sangaroon, W Ratanatongchai, R Picha, S Khaweerat, dan J. Channuie, 2017, The MCNP Simulation of a PGNAA System at TRR 1/M1, OP Conf. Series: Journal of Physics: Conf. Series 860
[4] Sutondo Tegas, Syarip, Beams Characteristic at radial and tangential beam ports of the KARTINI reactor, J. Iptek Nuklir Ganendra Ganendra, Vol. 17 No. 2: 83-90 (in Indonesian)

[5] Pelowitz, D.B., 2008, "MCNPX User's Manual Version 2.6.0", LANL, USA

[6] Niita, K. T. Sato, H. Iwase, H. Nose, H. Nakashima, L. Sihver, 2006, "PHITS-a particle and heavy ion transport code system," Radiat. Meas., vol. 41, pp. 1080-1090,

[7] Harmon C.D., Robert D.B., Judith F. Briesmeister, R.A. Forster, 1994, Criticality Calculations with $M C N P^{T M}$; A Primer, LA12827-M, Los Alamos National Laboratory, Los Alamos, New Mexico.

[8] Blakeman E.D., D.E. Peplow, J.C. Wagner, B.D. Murphy, D.E. Mueller, 2007, PWR Facility Dose Modeling Using MCNP5 and The CADIS/ADVANTG Variance-Reduction Methodology, ORNL/TM-2007/133, Oak Ridge National Laboratory. 